study. The program was delivered to $183 / 190$ (96\%) of drivers allocated to the intervention. In vehicle monitoring data was available for 351 participants (92\%) for a median of 52 weeks [inter-quartile range (IQR) 44-52] and 5487 [IQR 3294-8641] $\mathrm{km}$ of travel. $218 / 351$ (62\%) drivers had at least one RDE and the median number of RDEs was 1 [IQR $0-4]$. There was no between group difference in the rate of RDEs per distance driven (incident rate ratio (IRR) $0.85,95 \%$ CI 0.61-1.18). Crashes were reported by 14 participants in the intervention and 19 in the control group $(\mathrm{p}=0.46)$. Pre-planned sub-group analyses showed that the intervention was effective in significantly reducing RDEs (IRR 0.41, 95\% CI 0.20-0.81), in drivers with a DriveSafe/DriveAware score of 96 or higher (fit to continue driving).

Conclusions Older drivers with good visual and cognitive function are responsive to a one-on-one education program to improve their safety on the road. These drivers reduced their involvement in RDE events by more than half, however this approach was not effective in drivers with poorer function.

\section{OLDER PEOPLE, ALCOHOL AND SAFETY ISSUES AT HOME - THE PERSPECTIVE OF HOME CARE WORKERS}

Riitta Koivula, Anni Vilkko, Christoffer Tigerstedt, Kristiina Kuussaari, Satu Pajala. National Institute for Health and Welfare, Finland

\subsection{6/injuryprev-2016-042156.171}

Background Finland is a rapidly ageing society. The Finnish ageing policy prioritises older people living at home. Alcohol use of older people has become more common. Heavy drinking in particular leads to the need of increasing daily assistance and care. This paper discusses the challenging social situations of daily work of home care workers visiting alcohol consuming older people in their homes. This study, called "Ageing, alcohol and needs for services in home care" (2014-2015), is a part of the larger project "Harms to others from drinking: effects on health, wellbeing and the burden to society".

Methods The data consists of 13 thematic interviews with home care workers $(n=10)$ and their supervisors $(n=3)$. The interviews were conducted in one home care district in the metropolitan area in Finland.

Results When making their home visits home care workers have to handle challenging safety issues, such as alcohol-drug interactions, injury prevention and their own safety at work. Home care workers encounter unpredictable situations where they for safety reasons cannot fulfil their professional intentions.

Conclusions Home care workers are in need of further training about special skills to manage alcohol-related safety questions. Collaboration crossing professional and sectoral boundaries should be created to help those older people who are at the risk of alcohol-related safety issues. Further research is needed on older peoples' alcohol use from the point of view of home care workers.

\section{NEIGHBOURHOOD SAFETY AND INJURY PREVENTION AMONG OLDER ADULTS: A SYSTEMATIC LITERATURE REVIEW}

${ }^{1}$ Samuel N Forjuoh, ${ }^{2}$ Jaewoong Won, ${ }^{3}$ Marcia G Ory, ${ }^{2}$ Chanam Lee. ${ }^{1}$ Baylor Scott and White Health, Texas AandM HSC College of Medicine, USA; ${ }^{2}$ Texas AandM University, USA; ${ }^{3}$ Texas AandM HSC School of Public Health, USA

10.1136/injuryprev-2016-042156.172
Background Neighbourhood safety is important for older adults' health, including injury prevention and safety promotion, but there is a dearth of information about this construct in the literature.

Methods During 2014, we conducted a systematic literature review on the associations among identifiable neighbourhood safety factors, health outcomes, and health behaviours of older adults $(\geq 50)$ in the U.S. using MEDLINE, CINAHL, Embase, SportDis, and Transportation Databases.

Results Of 32 articles identified for our final review, 16 (50\%) examined health outcomes, such as health status and the other 16 focused on health behaviours, such as physical activity. Five domains of neighbourhood safety were identified: general neighbourhood safety; crime-related safety; traffic-related safety; fallrelated safety; and proxies for safety (e.g., vandalism, graffiti). Although falls are the leading cause of injuries in older adults, fall-related safety was the least frequently addressed safety domain. General neighbourhood safety, traffic-related safety, and proxies for safety appeared most relevant to health behaviours, while crime-related safety was most pertinent to health outcomes, such as mental health and physical function. Traffic-related safety showed more consistent associations for physical activity, while crime-related safety was more consistently associated with walking. We also found that specific measures or constructs of safety were not applied consistently across the different studies making it difficult to compare study findings.

Conclusions This review identified several patterns as well as many important gaps in the existing studies dealing with neighbourhood safety-injury prevention among older adults. We recommend that multi-dimensional neighbourhood safety factors should be considered in establishing location interventions, particularly related to injury prevention and safety promotion, which require further attention in future studies in the U.S. as well as globally.

\section{SEAT BELT FIT AND USE BEHAVIOURS OBSERVED AMONG DRIVERS AGED 75+ YEARS IN THEIR OWN VEHICLES}

${ }^{1}$ Julie Brown, ${ }^{2}$ Kristy Coxon, ${ }^{1}$ Cameron Fong, ${ }^{3}$ Elizabeth Clarke, ${ }^{1}$ Kris Rogers, ${ }^{2}$ Lisa Keay. ${ }^{1}$ Neuroscience Research Australia, and The University of New South Wales, Sydney, NSW, Australia; ${ }^{2}$ The George Institute for Global Health, The University of Sydney, Sydney, NSW, Australia; ${ }^{3}$ Kolling Institute of Medical Research, The University of Sydney, Sydney, NSW, Australia

\subsection{6/injuryprev-2016-042156.173}

Background Older occupants are at increased risk of serious injury in a crash compared to younger occupants. While seatbelts reduce injury risk, effectiveness relies on good belt fit and positioning. Laboratory research indicates increased likelihood of poor belt fit with increasing age, however little is known about seat belt use in this age group. The aims of this work are to (i) describe belt fit and use among drivers aged $75+$ years in their own vehicles, and (ii) examine the influence of body mass index (BMI), comfort and comorbidities on belt fit and use.

Methods Photographs were taken of drivers in their vehicles to assess belt fit and accessory use. Surveys of comorbidities and belt use, and measurements of height and weight were made. Logistic regression and mediation analysis examined associations between BMI, comfort, comorbidities and belt fit, belt repositioning behaviour, and accessory use.

Results 367 drivers were photographed, mean age 80 years, $23 \%$ used an accessory, $47 \%$ had poor sash and $41 \%$ poor lap 